\title{
Modern gastrointestinal endoscopic techniques for biliary tract cancers
}

\author{
Osman Ahmed, Jeffrey H. Lee \\ Department of Gastroenterology, Hepatology and Nutrition, University of Texas MD Anderson Cancer Center, Houston, TX, USA \\ Contributions: (I) Conception and design: All authors; (II) Administrative support: All authors; (III) Provision of study materials or patients: O Ahmed; \\ (IV) Collection and assembly of data: O Ahmed; (V) Data analysis and interpretation: O Ahmed; (VI) Manuscript writing: All authors; (VII) Final \\ approval of manuscript: All authors. \\ Correspondence to: Jeffrey H. Lee, MD, MPH, FASGE, FACG, AGAF. Professor and Medical Director of Endoscopy Center, Department of \\ Gastroenterology, Hepatology and Nutrition, University of Texas MD Anderson Cancer Center, 1515 Holcombe Avenue, Houston, TX 77030, \\ USA. Email: jefflee@mdanderson.org.
}

\begin{abstract}
Biliary tract cancers, specifically cholangiocarcinomas (CCAs), arise from the epithelial cells of the biliary tree. They can be divided into three groups based on their location: intra-hepatic, peri-hilar or distal extra-hepatic CCAs. Traditionally, the main role of endoscopy in the management of biliary tract cancers was diagnosis and biliary decompression. For diagnosis, endoscopic retrograde cholangiopancreatography (ERCP) can be used to obtain either brushings or intra-ductal biopsies however both techniques have poor sensitivity. The introduction of cholangioscopy has allowed endoscopists to perform both targeted biopsies and also obtain a visual diagnosis. Similarly, with the spread of endoscopic ultrasound (EUS), the ability to obtain tissue by fine-needle aspiration is another avenue available, but concerns regarding tumor seeding still persist. For biliary decompression, with the advent of neo-adjuvant therapy, the role of early decompression is growing. Nevertheless, it is still not clear whether endoscopic decompression is superior to percutaneous decompression, especially in advanced hilar tumors. When possible, at least $50 \%$ of viable liver should be drained, and that will determine whether unilateral or bilateral stents are required. Additionally, there is growing evidence on the benefits of metal stents over plastic stents, but care should be taken as metal stents are generally permanent. Finally, although not widely available or adopted, with the growing use of radiofrequency ablation and the introduction of drug-eluting metal stents, the near-future might allow newer techniques to treat the disease itself.
\end{abstract}

Keywords: Cholangiocarcinoma (CCA); endoscopy; stents; biliary; diagnosis

Submitted Jul 06, 2019. Accepted for publication Sep 27, 2019.

doi: $10.21037 /$ cco.2019.09.04

View this article at: http://dx.doi.org/10.21037/cco.2019.09.04

\section{Introduction}

Biliary tract cancers, specifically cholangiocarcinomas (CCAs), are cancers that arise within the biliary tree. CCAs generally arise from the epithelial cells and are the second most common primary liver malignancies encountered. They account for approximately $3 \%$ of all gastrointestinal malignancies and roughly $15 \%$ of all hepatobiliary malignancies (1).

CCAs can be divided into three separate categories based on their anatomy; intra-hepatic CCAs (iCCAs), hilar or peri-hilar CCAs (also known as Klatskin tumors), and distal extra-hepatic CCAs. iCCAs arise above the secondorder branches of the biliary tree, whereas the two extrahepatic malignancies are generally divided by the cystic duct take-off (Figure 1) (2). More than two-thirds of CCAs are extra-hepatic; with peri-hilar tumors accounting for $\sim 50 \%$, and intra-hepatic tumors only contributing $\sim 10 \%$ of all CCAs (3). 


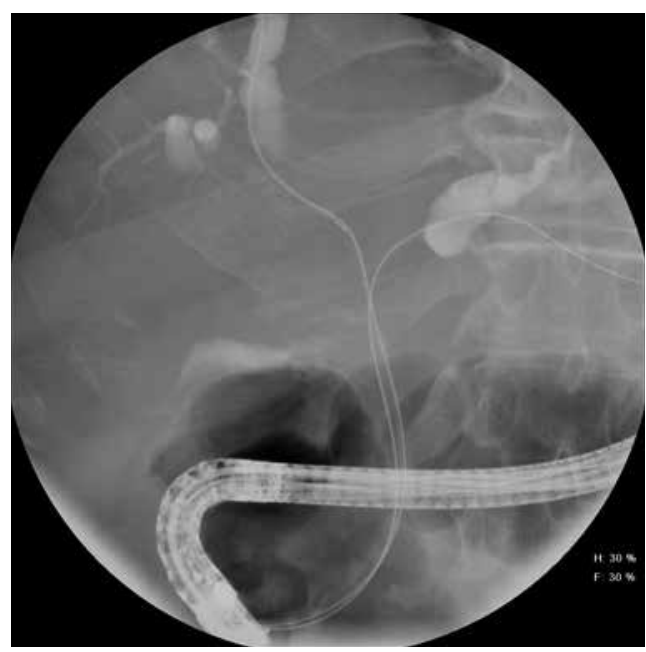

Figure 1 Hilar CCA. CCA, cholangiocarcinoma.

CCAs have a wide range of incidences geographically, with the most common locations being in East Asia, likely due to differences in genetic and environmental factors. Overall, the incidence of CCAs continues to rise, with the largest rise being amongst iCCAs. Within the United States, the incidence of iCCAs has been increasing by $2.3 \%$ per year over the last forty years, whereas the rate of extra-hepatic CCAs has largely been stable $(4,5)$. There are many risk factors that have been identified to potentially contribute to the development of CCAs and explain the recent rise in incidence including inflammatory bowel disease, cirrhosis, choledochal cyst, diabetes, thyrotoxicosis and chronic pancreatitis. In regards to iCCAs specifically; smoking, hepatitis $\mathrm{C}$ infection and non-alcoholic liver disease are thought to be risk factors $(6,7)$. Nevertheless, the majority of patients do not have an identifiable risk factor at diagnosis (8).

The endoscopic approach to CCA has traditionally been two-prong; the first was to assist in the diagnosis of the disease, and the second was to relieve any biliary obstruction. Nonetheless, the emergence of newer endoscopic modalities, and the development of novel instruments within endoscopy has now led to a renewed interest in using endoscopy for new indications in the management of the disease and its complications.

In this review, we will explore the role of endoscopy in the diagnosis of biliary tract cancers, from endoscopic retrograde cholangiopancreatography (ERCP)-based tools to endoscopic ultrasound (EUS)-guided tissue acquisition. We will also explore the controversies in the management of biliary obstructions, especially in regards to when, how and where to drain the bile ducts. Finally, we will explore newer techniques and specific situations that can potentially allow for the treatment of the disease itself.

\section{Diagnosis}

The diagnosis of CCAs can be challenging not only due to the difficulty in obtaining tissue sampling, but also due to the lack of evidence-based screening protocols, and the fact that symptoms generally do not present until later in the disease. The most common presentation in patients with CCA is the development of jaundice. However, this only occurs in about $10-15 \%$ of patients when they initially present. The presentation of jaundice is related to the development of biliary obstruction, which leads to an increase in bilirubin levels. Other common symptoms that occur in patients with CCA are generally non-specific and include, amongst others, abdominal pain, weight loss and general malaise. In approximately $20-25 \%$ of patients, the finding of CCA is discovered incidentally $(9,10)$.

The ability to obtain tissue-sampling is another dilemma that is faced by physicians in managing CCA. Because definitive treatment options often come associated with increased morbidity, and in some cases, mortality, the ability to confirm diagnosis would be ideal. However, because of a variety of reasons, including location, histology, and current available investigative methods, that is not always possible. In a retrospective study, roughly $40 \%$ of patients sent to surgery for presumed CCA did not have a prior tissue confirmation, and roughly $10 \%$ of patients who did undergo surgery, did not have evidence of any malignancy in their resected specimen (11).

ERCP is a common technique used for the diagnosis of CCAs. Because ERCP allows for the concomitant ability to drain the biliary tree, it is seen advantageous as it allows for one procedure to accomplish both diagnosis and drainage. The simplest method to obtain cells for diagnosis is through the use of brushings. In this method, the epithelium of the bile ducts is brushed using a catheter which has bristles on its sides that allow it to obtain cells. The specimen is then viewed by a cytologist to see if any malignant cells are present. Although simple to perform, brushings are limited by their low sensitivity. In two previous meta-analysis, the sensitivity of brushings was approximately $45 \%$, however the specificity of brushings was close to $99 \%$ (12-14).

Another method of sampling the bile duct during ERCP is to obtain intra-ductal biopsy specimens. Intra- 


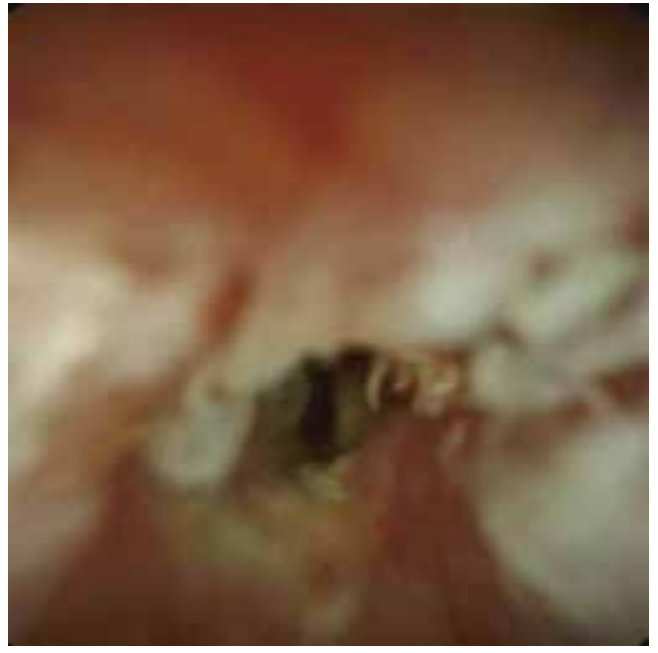

Figure 2 Cholangioscopy of CCA. CCA, cholangiocarcinoma.

ductal biopsies can be obtained using either wire-guided biopsy forceps, regular forceps or pediatric forceps with a combination of fluoroscopy to determine the location of the stricture. Biopsy specimens are generally placed in formalin to be analyzed by a pathologist and allow for a larger sampling size compared to brushings. Nevertheless, similar to brushings, a recent meta-analysis of nine studies demonstrated that the yield of intra-ductal biopsies was low, with a sensitivity less than $50 \%$, but a specificity also around $~ 99 \%$ (12). The idea of performing both brushings and intra-ductal biopsies has also been studied, with the thought that the combination of the two would improve the sensitivity over either one individually. Similarly, since they are both performed during ERCP, it would not require another procedure, and would only add a minimal amount of additional time to the procedure. Nonetheless, the combination of brushings and intra-ductal biopsies only increased the sensitivity to $59 \%$, with a specificity of $100 \%$ to detect malignant biliary strictures (12).

Although ERCP alone with brushings and/or intraductal biopsies did not have a significant ability to obtain histological or cytological confirmation, it does allow the ability to perform cholangioscopy, where a smaller probe is inserted into the bile ducts and allows for optical visualization of the biliary tree (Figure 2). The introduction of single-operator cholangioscopes and improved visualization have led to an improvement in the ability to investigate indeterminate strictures. There are two methods of diagnosis with cholangioscopy: direct visualizationimpression diagnosis and the ability to obtain targeted biopsies for histological diagnosis. For visual impression, a previous meta-analysis involving 8 studies and 335 patients demonstrated a sensitivity of $90 \%$ with a specificity of $80 \%$ (15). In regards to targeted tissue biopsies, a metaanalysis of 10 studies involving 456 patients showed that the sensitivity of cholangioscopy was $60 \%$ with a specificity of 98\% (16).

Aside from ERCP, there has been a growing interest in utilizing EUS to obtain the diagnosis in patients with indeterminate biliary strictures. The advantage of EUS is the ability to perform fine-needle aspiration to obtain a cytological diagnosis. Previous studies have demonstrated a pooled sensitivity of up to $80 \%$ when a mass lesion is seen on EUS, including a sensitivity of almost $60 \%$ in patients who previously had negative brush biopsies $(17,18)$. The main concern in regards to EUS guided fine needle aspiration (EUS-FNA) is the potential of tumor seeding as the needle crosses the peritoneum when obtaining a sample. An older study demonstrated higher rates of disseminated disease in patients who underwent trans-peritoneal FNA followed by liver transplant compared to those who did not undergo FNA. Due to this, some institutions will exclude patients for liver transplant if they have undergone FNA (19). Patients with distal extra-hepatic CCA however can generally undergo FNA, as the planned surgery (Whipple's resection) would include removing part of the duodenum and therefore the risk of seeding is less of a concern.

Overall, no one technique has shown to be sufficient in the diagnosis of CCA, and the best approach is a combination of two or more methods depending on physician expertise, local availability and the patient's tumor characteristics.

\section{Biliary decompression}

Because biliary tract cancers grow within the bile ducts, which are generally less than a centimeter in diameter, they can quickly cause obstruction of bile drainage and result in jaundice. The necessity to decompress the bile ducts is vital, especially if there are plans to provide any chemotherapy, including in the neo-adjuvant setting.

Although biliary decompression is essential, there is controversy on whether patients who are planned to undergo surgical resection should undergo endoscopic and/ or percutaneous drainage prior to surgery or whether they should be referred directly to surgery. A study examining 905 patients who underwent surgical resection for proximal (hilar or intra-hepatic) CCAs found an increased rate of 


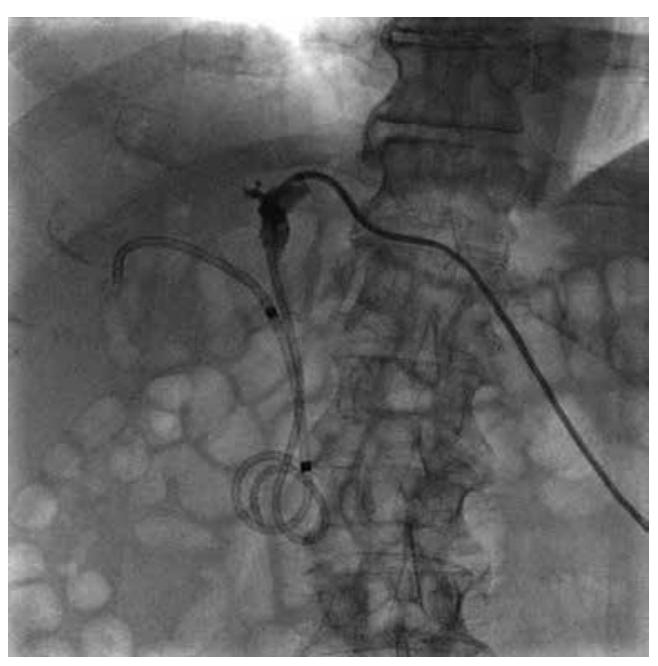

Figure 3 Percutaneous and endoscopic stent placement.

post-operative complications in those who underwent biliary drainage prior to surgery, including an increased rate of infections, liver failure and mortality (20). A separate study from Europe, examined 366 patients with only hilar disease and found no differences in outcomes after surgical resection in those who underwent pre-operative drainage and those that did not (21). Finally, a retrospective database study involving 3,862 patient with extra-hepatic CCA who underwent surgery found shortened survival time in patients who underwent pre-operative biliary drainage (22).

Nevertheless, although the evidence suggests that preoperative biliary drainage might lead to worse outcomes, the growing use of neo-adjuvant therapy to improve outcomes limits the ability to hasten surgery. In those patients, it is imperative to obtain biliary decompression in order to decrease bilirubin levels so that chemotherapeutic agents can be given. Similarly, patients who need optimization (e.g., malnutrition) or those patients who present with cholangitis should receive prompt decompression. Overall, previous studies have shown that patients who successfully undergo biliary decompression when indicated have improved mortality compared to those who do not (23).

\section{Endoscopic or percutaneous drainage}

In patients who will undergo biliary drainage, the next step is determining the best method to pursue. Traditionally, there have been two methods to achieve biliary decompression; the endoscopic method and the percutaneous method. The endoscopic method is generally achieved by performing an ERCP, and as mentioned earlier, can be useful to obtain tissue samples as well. It requires the patient to undergo an endoscopy to the ampulla (usually with sedation), and then successfully cannulate to gain access to the biliary tree. The main benefit of ERCP is that all drainage is done internally. In addition, with the newly developed EUS-guided approaches of hepaticogastrostomy, choledochoduodenostomy, and rendez-vous techniques, the technical success rate of endoscopic methods has reached a new high. In the percutaneous method, also known as percutaneous trans-hepatic cholangiography (PTC), the catheter is advanced through the skin, into a branch of the bile duct that is intra-hepatic to gain access to the biliary tree (Figure 3). The advantage is that it does not require endoscopy to perform, however, it generally does require external drainage for some amount of time. Finally, although surgical biliary drainage through the formation of a biliary-enteric anastomosis was performed in the past, its use has fallen out of favor as the two previously described techniques have become widely available (24).

The evidence comparing endoscopic to percutaneous biliary drainage has still not validated one technique as being superior. One of the earliest studies comparing the two methods demonstrated that the endoscopic approach had a higher success rate and a lower mortality compared to PTC (25). However, subsequent studies have been more equivocal, including a recent study comparing 106 consecutive patients undergoing biliary drainage with CCA. Although the success rate was similar, the endoscopic group had a higher rate of complications (mainly cholangitis and pancreatitis), though the complications were able to be managed without long-term sequelae, whereas in the PTC group, two patients developed disseminated cancer caused by catheter insertion (26).

A systematic review and meta-analysis, comparing ERCP to PTC included 15 studies and found no difference in success rates, but a higher peri-procedural complication rate with endoscopic drainage, even though long-term outcomes were similar (27). A different meta-analysis, of only four studies, looking only at patients with peri-hilar tumors that were resectable candidates, found that PTC had higher success rates and lower peri-procedural complications (28).

Overall, due to the lack of consensus currently on the optimal drainage method, the choice of how to proceed with decompression ultimately lies with a variety of factors; including physician expertise, patient preference and tumor characteristics (29). Nevertheless, an upcoming randomized controlled trial comparing the two modalities is eagerly 
awaited to see if any one modality is preferable to the other (30).

\section{Unilateral or bilateral stent}

The next decision to be made, once the timing and mechanism of biliary decompression is decided, is how much of the liver to drain. The liver generally has two large lobes that can be drained. Although there are variants in the anatomy, there are generally three first-order branch ducts that can be drained, the right anterior hepatic duct, the right posterior hepatic duct, and the left hepatic duct.

The question of unilateral $v s$. bilateral drainage generally lies in terms of accessible ducts and viable liver. A previous study demonstrated that as long as at least $50 \%$ of the liver was being drained, the bilirubin of a patient should normalize and lead to sufficient drainage. Similarly, drainage of any atrophic part of the liver was harmful, as not only did it not improve the patient's biochemical profile, but also increased the risk of cholangitis (31). The determination of how much liver volume to be drained is generally done by estimating based on viable liver seen on cross-sectional imaging. A more recent study has suggested that potentially only a third of the liver would need to be drained to obtain appropriate decompression if there were no signs of liver dysfunction, while $50 \%$ of the liver should be drained in patients with liver dysfunction (32).

In terms of placement of stents, a previous meta-analysis of 10 studies showed that unilateral drainage had a higher rate of successful stent insertion, with no difference in successful drainage, complications or stent patency (33). A more recent randomized controlled trial of 133 patients, showed similar technical success rates but fewer reinterventions and longer stent patency with bilateral stents. The only limitation was that the study only utilized selfexpanding metal stents in their comparison (34).

Although the decision about unilateral vs. bilateral drainage ultimately comes down to the location of the stricture, generally the location of strictures is determined using a combination of opacifying the bile ducts using contrast dye and fluoroscopy. However, obtaining and reviewing a cross-sectional imaging study prior to biliary drainage procedure is paramount, in order to prevent cholangitis. The caveat to this is that is it critical that only ducts and lobes that can be drained by injected with contrast, as undrained lobes that have been injected are more likely to cause cholangitis. A seminal study from Canada demonstrated that when compared with patients where all ducts injected were drained, patients who had ducts that were injected but left undrained had higher mortality rates (35).

\section{Metal or plastic stent}

There are generally two types of stents that can be placed in patients with biliary tract cancers: plastic polyethylenebased stents and self-expanding metal stents that are usually nitinol-based. The decision to place a plastic or metal stent is usually made based on the location of the tumor. Of note, patients who are potential surgical candidates, should either have plastic stents placed, or be seen by a surgeon prior to placement of any self-expandable metal stent (SEMS), especially if uncovered, as those stents cannot be removed.

In patients with unresectable hilar CCAs, the evidence to place a metal stent over a plastic stent is growing. The initial retrospective study examining plastic to metal stents was a retrospective study of 100 patients with inoperable CCA. The results showed that metal stent placements had longer patency, less re-interventions and no differences in complications compared to plastic stents (36). A subsequent larger retrospective date of 450 patients showed similar results with higher patency, higher technical and clinical success and less complications with metal stents (37).

More recently, a randomized study of 108 patients with hilar CCA showed that metal stent placement provided better adequacy of drainage and longer survival compared to plastic stents. The median survival time was 126 days in the SEMS group, and 49 days in the plastic stent group (38).

In regards to bilateral placement of metal stents, they can be placed in two configurations: side-by-side placement ("double-barrel" stent placement) or stent-in-stent placement ("Y-configuration" stent placement). The benefit of stent-in-stent is that it does not require a dilated common hepatic duct to fit, and therefore theoretically larger stents can be placed (Figure 4). The advantage of side-by-side placement is that it generally is technically easier to place and easier to access if any revision is required in the future (Figures 5,6). Nevertheless, the largest study comparing the two approaches included 69 patients randomized to either configuration. The study demonstrated no difference between the two in terms of stent patency, technical success, clinical success or overall survival (39). An older study of 52 consecutive patients however demonstrated higher complications with side-by-side placement but longer patency as compared to stent-in-stent placement (40).

The benefit of plastic stents is that they are removable, 


\section{Page 6 of 9}

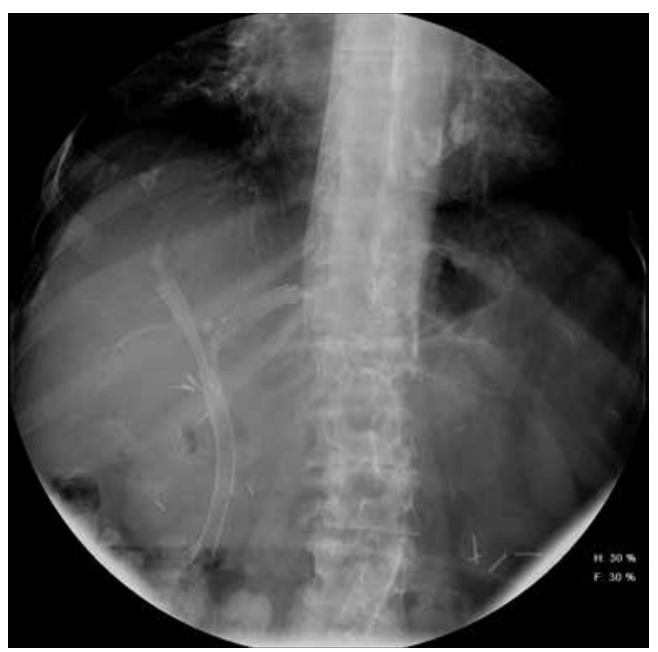

Figure 4 Stent-in-stent SEMS insertion. SEMS, self-expandable metal stent.

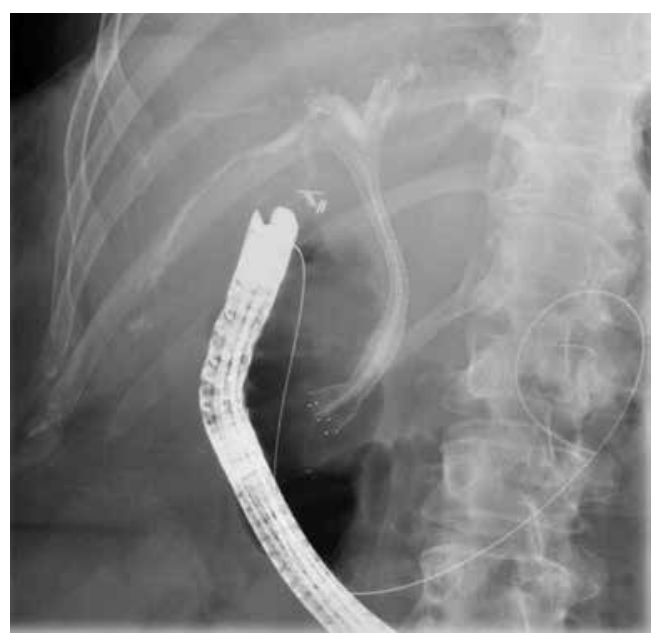

Figure 5 Side-by-side SEMS insertion. SEMS, self-expandable metal stent.

cost-less and can be placed in multiple segments without difficulty. The main disadvantage is that due to their smaller diameter, they require prophylactic stent exchanges to prevent obstruction. The most common size of plastic stents are 7 and 10 French in diameter. Self-expanding metal stents come in a variety of sizes, from 6 to $10 \mathrm{~mm}$, and can be either uncovered, partially covered or fully covered. The advantage of SEMS is that they generally show longer patency, with the disadvantage being higher cost, and if uncovered, the inability to remove or exchange if needed. In

\section{Ahmed and Lee. Endoscopic approach of biliary malignancy}

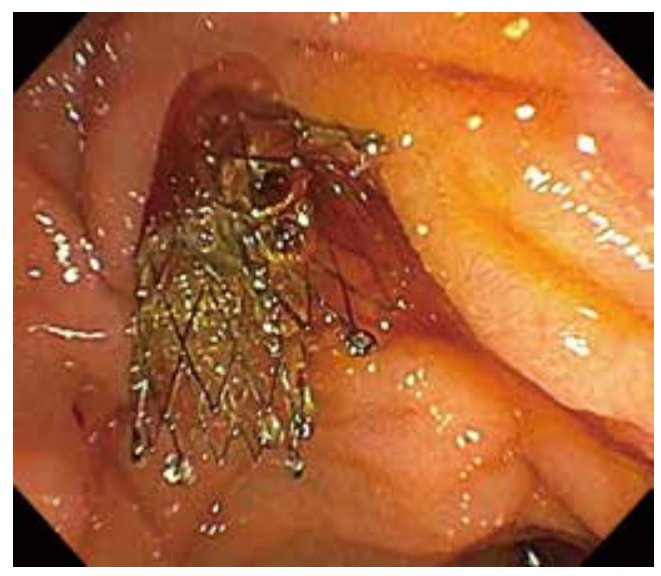

Figure 6 Endoscopic view of side-by-side SEMS insertion. SEMS, self-expandable metal stent.

addition, if second order branches are involved, metal stents may occlude them increasing the risk of cholangitis and/or atrophy of the liver.

\section{Novel therapeutics}

Although, the cornerstone of endoscopic techniques for CCA has been diagnosis and biliary decompression, the ongoing growth of the field has allowed for the development of newer techniques that attempt to not only decrease the rates of re-intervention but potentially also treat the disease.

One of the more rapidly developing modalities is the use of radiofrequency ablation in the management of extrahepatic CCAs. In this technique, a bipolar catheter is advanced to the desired area of stenosis, and thermal energy is applied to deliver coagulation necrosis to the tissue. Although, widely studied and used in the esophagus in patients with Barrett's esophagus, the concept has now been adopted for malignant biliary strictures (41).

The initial proof-of-concept study was published in 2011 and showed successful ablation with stent placement in 21 out of 22 patients, with only one episode of pancreatitis (42). A subsequent larger study of 64 patients with malignant biliary strictures compared stent placement alone with radiofrequenzablation (RFA) combined with stent placement and found no difference with patency but increased overall survival in the RFA group (43). A separate retrospectively compared patients with CCA who had undergone photodynamic therapy (PDT) to RFA. They found that there was no difference in survival between the two groups, 
but patients who had undergone RFA had a lower number of stents placed but a higher rate of developing cholangitis (44).

A more recent study examined the role of performing ERCP combined with RFA in operable candidates who require pre-operative drainage found that RFA was successful in all 8 patients. Examination of the resected tissue showed a maximal ablation depth of 4 (range, $1-6) \mathrm{mm}$ and roughly $72 \%$ of the malignant stricture was successfully ablated with RFA (45). Of note, in a study involving 12 patients (19 RFA sessions), biliary bleeding was observed in 3 patients, 4-6 weeks after RFA; unfortunately, two of the 3 patients died from hemorrhagic shock (46).

Another developing therapeutic technique is the insertion of a drug-eluting self-expanding metal stent to reduce the rates of stent occlusion and re-intervention. The initial studies have mainly examined using paclitaxel-eluting metal stents, but recently gemcitabine and sorafenib-eluting stents were also studied in porcine models (47-49). Nevertheless, there is a paucity of data for drug-eluting stents in human studies. Two previous human studies comparing paclitaxeleluting metal stents to standard metal stents found no difference in stent patency or survival but did note that the safety of drug-eluting stents was similar to traditional stents $(50,51)$.

Overall, although the novel endoscopic techniques may not be ready for widespread adoption, they do suggest a burgeoning role for endoscopy outside of the traditional diagnosis and biliary decompression aspects.

\section{Conclusions}

Over the last few decades, the role of endoscopy has drastically changed, not only in terms of luminal disease, but also pancreatobiliary diseases. The development of ERCP allowed for access into the biliary tree, and the development of brushings and biopsy forceps allowed for sampling of biliary strictures. Subsequently, the development of plastic and then self-expanding metal stents allowed endoscopic internal biliary drainage. Concurrently with the development of ERCP, was the growing use of EUS, including the development of FNA techniques for sampling. Finally, the recent expansion of cholangioscopy has further altered our approach to biliary disease, and upcoming tools such as radio-frequency ablation and new modes of stent delivery and type, promise to further change our approach to endoscopic management of CCAs.

\section{Acknowledgments}

None.

\section{Footnote}

Conflicts of Interest: The authors have no conflicts of interest to declare.

Etbical Statement: The authors are accountable for all aspects of the work in ensuring that questions related to the accuracy or integrity of any part of the work are appropriately investigated and resolved.

\section{References}

1. Bergquist A, von Seth E. Epidemiology of cholangiocarcinoma. Best Pract Res Clin Gastroenterol 2015;29:221-32.

2. Rizvi S, Khan SA, Hallemeier CL, et al. Cholangiocarcinoma - evolving concepts and therapeutic strategies. Nat Rev Clin Oncol 2018;15:95-111.

3. Sharma P, Yadav S. Demographics, tumor characteristics, treatment, and survival of patients with Klatskin tumors. Ann Gastroenterol 2018;31:231-6.

4. Saha SK, Zhu AX, Fuchs CS, et al. Forty-year trends in cholangiocarcinoma incidence in the U.S.: intrahepatic disease on the rise. Oncologist 2016;21:594-9.

5. Shaib Y, El-Serag HB. The epidemiology of cholangiocarcinoma. Semin Liver Dis 2004;24:115-25.

6. Welzel TM, Graubard BI, El-Serag HB, et al. Risk factors for intrahepatic and extrahepatic cholangiocarcinoma in the United States: a population-based case-control study. Clin Gastroenterol Hepatol 2007;5:1221-8.

7. Petrick JL, Yang B, Altekruse SF, et al. Risk factors for intrahepatic and extrahepatic cholangiocarcinoma in the United States: a population-based study in SEERMedicare. PLoS One 2017;12:e0186643.

8. Banales JM, Cardinale V, Carpino G, et al. Expert consensus document: Cholangiocarcinoma: current knowledge and future perspectives consensus statement from the European Network for the Study of Cholangiocarcinoma (ENS-CCA). Nat Rev Gastroenterol Hepatol 2016;13:261-80.

9. Endo I, Gonen M, Yopp AC, et al. Intrahepatic cholangiocarcinoma: rising frequency, improved survival, and determinants of outcome after resection. Ann Surg 2008;248:84-96. 
10. Alvaro D, Bragazzi MC, Benedetti A, et al.

Cholangiocarcinoma in Italy: a national survey on clinical characteristics, diagnostic modalities and treatment.

Results from the "cholangiocarcinoma" committee of the Italian Association for the study of liver disease. Dig Liver Dis 2011;43:60-5.

11. Nuzzo G, Giuliante F, Ardito F, et al. Improvement in perioperative and long-term outcome after surgical treatment of hilar cholangiocarcinoma: results of an Italian multicenter analysis of 440 patients. Arch Surg 2012;147:26-34.

12. Navaneethan U, Njei B, Lourdusamy V, et al. Comparative effectiveness of biliary brush cytology and intraductal biopsy for detection of malignant biliary strictures: a systematic review and meta-analysis. Gastrointest Endosc 2015;81:168-76.

13. Trikudanathan G, Navaneethan U, Njei B, et al. Diagnostic yield of bile duct brushings for cholangiocarcinoma in primary sclerosing cholangitis: a systematic review and meta-analysis. Gastrointest Endosc 2014;79:783-9.

14. Burnett AS, Calvert TJ, Chokshi RJ. Sensitivity of endoscopic retrograde cholangiopancreatography standard cytology: 10-y review of the literature. J Surg Res 2013;184:304-11.

15. Sun $X, Z$ hou Z, Tian J, et al. Is single-operator peroral cholangioscopy a useful tool for the diagnosis of indeterminate biliary lesion? A systematic review and meta-analysis. Gastrointest Endosc 2015;82:79-87.

16. Navaneethan U, Hasan MK, Lourdusamy V, et al. Singleoperator cholangioscopy and targeted biopsies in the diagnosis of indeterminate biliary strictures: a systematic review. Gastrointest Endosc 2015;82:608-14.e2 .

17. Navaneethan U, Njei B, Venkatesh PG, et al. Endoscopic ultrasound in the diagnosis of cholangiocarcinoma as the etiology of biliary strictures: a systematic review and metaanalysis. Gastroenterol Rep (Oxf) 2015;3:209-15.

18. Sadeghi A, Mohamadnejad M, Islami F, et al. Diagnostic yield of EUS-guided FNA for malignant biliary stricture: a systematic review and meta-analysis. Gastrointest Endosc 2016;83:290-8.e1.

19. Heimbach JK, Sanchez W, Rosen CB, et al. Transperitoneal fine needle aspiration biopsy of hilar cholangiocarcinoma is associated with disease dissemination. HPB (Oxford) 2011;13:356-60.

20. Ramanathan R, Borrebach J, Tohme S, et al. Preoperative biliary drainage is associated with increased complications after liver resection for proximal cholangiocarcinoma. J Gastrointest Surg 2018;22:1950-7.
21. Farges O, Regimbeau JM, Fuks D, et al. Multicentre European study of preoperative biliary drainage for hilar cholangiocarcinoma. Br J Surg 2013;100:274-83.

22. Navaneethan U, Zhu X, Parsi MA, et al. Pre-operative biliary drainage is associated with shortened survival time in patients with cholangiocarcinoma. Gastroenterol Rep (Oxf) 2019;7:185-92.

23. Cassani LS, Chouhan J, Chan C, et al. Biliary decompression in perihilar cholangiocarcinoma improves survival: a single-center retrospective analysis. Dig Dis Sci 2019;64:561-9.

24. Smith AC, Dowsett JF, Russell RC, et al. Randomised trial of endoscopic stenting versus surgical bypass in malignant low bileduct obstruction. Lancet 1994;344:1655-60.

25. Speer AG, Cotton PB, Russell RC, et al. Randomised trial of endoscopic versus percutaneous stent insertion in malignant obstructive jaundice. Lancet 1987;2:57-62.

26. Kim KM, Park JW, Lee JK, et al. A comparison of preoperative biliary drainage methods for perihilar cholangiocarcinoma: endoscopic versus percutaneous transhepatic biliary drainage. Gut Liver 2015;9:791-9.

27. Hameed A, Pang T, Chiou J, et al. Percutaneous vs. endoscopic pre-operative biliary drainage in hilar cholangiocarcinoma - a systematic review and metaanalysis. HPB (Oxford) 2016;18:400-10.

28. Al Mahjoub A, Menahem B, Fohlen A, et al. Preoperative biliary drainage in patients with resectable perihilar cholangiocarcinoma: is percutaneous transhepatic biliary drainage safer and more effective than endoscopic biliary drainage? A meta-analysis. J Vasc Interv Radiol 2017;28:576-82.

29. Duan F, Cui L, Bai Y, et al. Comparison of efficacy and complications of endoscopic and percutaneous biliary drainage in malignant obstructive jaundice: a systematic review and meta-analysis. Cancer Imaging 2017;17:27.

30. Al-Kawas F, Aslanian H, Baillie J, et al. Percutaneous transhepatic vs. endoscopic retrograde biliary drainage for suspected malignant hilar obstruction: study protocol for a randomized controlled trial. Trials 2018;19:108.

31. Vienne A, Hobeika E, Gouya H, et al. Prediction of drainage effectiveness during endoscopic stenting of malignant hilar strictures: the role of liver volume assessment. Gastrointest Endosc 2010;72:728-35.

32. Takahashi E, Fukasawa M, Sato T, et al. Biliary drainage strategy of unresectable malignant hilar strictures by computed tomography volumetry. World J Gastroenterol 2015;21:4946-53.

33. Hong W, Sun X, Zhu Q. Endoscopic stenting for 
malignant hilar biliary obstruction: should it be metal or plastic and unilateral or bilateral? Eur J Gastroenterol Hepatol 2013;25:1105-12.

34. Lee TH, Kim TH, Moon JH, et al. Bilateral versus unilateral placement of metal stents for inoperable highgrade malignant hilar biliary strictures: a multicenter, prospective, randomized study (with video). Gastrointest Endosc 2017;86:817-27.

35. Chang WH, Kortan P, Haber GB. Outcome in patients with bifurcation tumors who undergo unilateral versus bilateral hepatic duct drainage. Gastrointest Endosc 1998;47:354-62.

36. Raju RP, Jaganmohan SR, Ross WA, et al. Optimum palliation of inoperable hilar cholangiocarcinoma: comparative assessment of the efficacy of plastic and selfexpanding metal stents. Dig Dis Sci 2011;56:1557-64.

37. Liberato MJA, Canena JMT. Endoscopic stenting for hilar cholangiocarcinoma: efficacy of unilateral and bilateral placement of plastic and metal stents in a retrospective review of 480 patients. BMC Gastroenterol 2012;12:103.

38. Sangchan A, Kongkasame W, Pugkhem A, et al. Efficacy of metal and plastic stents in unresectable complex hilar cholangiocarcinoma: a randomized controlled trial. Gastrointest Endosc 2012;76:93-9.

39. Lee TH, Moon JH, Choi JH, et al. Prospective comparison of endoscopic bilateral stent-in-stent versus stent-by-stent deployment for inoperable advanced malignant hilar biliary stricture. Gastrointest Endosc 2019;90:222-30.

40. Naitoh I, Hayashi K, Nakazawa T, et al. Side-by-side versus stent-in-stent deployment in bilateral endoscopic metal stenting for malignant hilar biliary obstruction. Dig Dis Sci 2012;57:3279-85.

41. ASGE Technology Committee, Navaneethan U, Thosani $\mathrm{N}$, et al. Radiofrequency ablation devices. VideoGIE 2017;2:252-9.

42. Steel AW, Postgate AJ, Khorsandi S, et al. Endoscopically applied radiofrequency ablation appears to be safe in the treatment of malignant biliary obstruction. Gastrointest

Cite this article as: Ahmed O, Lee JH. Modern gastrointestinal endoscopic techniques for biliary tract cancers. Chin Clin Oncol 2020;9(1):3. doi: 10.21037/cco.2019.09.04
Endosc 2011;73:149-53.

43. Sharaiha RZ, Natov N, Glockenberg KS, et al. Comparison of metal stenting with radiofrequency ablation versus stenting alone for treating malignant biliary strictures: is there an added benefit? Dig Dis Sci 2014;59:3099-102.

44. Strand DS, Cosgrove ND, Patrie JT, et al. ERCP-directed radiofrequency ablation and photodynamic therapy are associated with comparable survival in the treatment of unresectable cholangiocarcinoma. Gastrointest Endosc 2014;80:794-804.

45. Kim EJ, Chung DH, Kim YJ, et al. Endobiliary radiofrequency ablation for distal extrahepatic cholangiocarcinoma: a clinicopathological study. PLoS One 2018; 13:e0206694.

46. Tal AO, Vermehren J, Friedrich-Rust M, et al. Intraductal endoscopic radiofrequency ablation for the treatment of hilar non-resectable malignant bile duct obstruction. World J Gastrointest Endosc 2014;6:13-9.

47. Jang SI, Kim JH, Kim M, et al. Porcine feasibility and safety study of a new paclitaxel-eluting biliary stent with a Pluronic-containing membrane. Endoscopy 2012;44:825-31.

48. Chung MJ, Kim H, Kim KS, et al. Safety evaluation of self-expanding metallic biliary stents eluting gemcitabine in a porcine model. J Gastroenterol Hepatol 2012;27:261-7.

49. Kim DH, Jeong YI, Chung CW, et al. Preclinical evaluation of sorafenib-eluting stent for suppression of human cholangiocarcinoma cells. Int J Nanomedicine 2013;8:1697-711.

50. Suk KT, Kim JW, Kim HS, et al. Human application of a metallic stent covered with a paclitaxel-incorporated membrane for malignant biliary obstruction: multicenter pilot study. Gastrointest Endosc 2007;66:798-803.

51. Jang SI, Kim JH, You JW, et al. Efficacy of a metallic stent covered with a paclitaxel-incorporated membrane versus a covered metal stent for malignant biliary obstruction: a prospective comparative study. Dig Dis Sci 2013;58:865-71. 